\title{
El derecho de propiedad comunal en el marco del Convenio 169 de la Organización Internacional del Trabajo (OIT) en las comunidades nativas del distrito de Chazuta, Provincia y Región de San Martin
}

The right of communal property within the framework of Convention 169 of the International Labor Organization (ILO) in the native communities of the district of Chazuta, Province and Region of San Martín

Cenepo Mozombite, Basilio' ${ }_{9110-4475]}^{1}$ Bardales del Aguila, Lionel ${ }^{1[0000-0002-}$

${ }^{1}$ Universidad Nacional de San Martín, Tarapoto, Perú lbardales@unsm.edu.pe

Resumen. El objetivo fue estudiar el derecho de la propiedad de las comunidades nativas del distrito de Chazuta, provincia y región de San Martín reconocido en el Convenio 169 de la Organización Internacional del Trabajo (OIT). La investigación es de tipo aplicada de enfoque mixto cuantitativo-cualitativo, el diseño no experimental de nivel descriptivo transversal, la población y muestra de estudio la conformaron 64 pobladores y 4 Apus de las comunidades nativas Kechwas de Mushukllacta de Chipaota, Shilcayo, Canayo y Llucanayacu; ubicadas en el distrito de Chazuta. Para recoger la información se utilizaron dos cuestionarios: Una encuesta para los pobladores y una entrevista estructurada para los Apus. Los resultados de las encuestas muestran que el $61 \%$ de los pobladores desconocen la cantidad de hectáreas pertenecientes al territorio comunal; el 39\% de encuestados reconocen que el Estado les brinda apoyo para la titulación de su territorio. Los Apus de las Comunidades de Shilcayo y Canayo, afirman que su territorio se encuentra concesionada y superpuesta al Área de Conservación Regional Cordillera Escalera y Bosque de Protección Nacional Cordillera Azul. Es evidente, que los rezagos del conflicto histórico por la ocupación y en muchos casos usurpación del territorio de los pueblos originarios aún persisten, por lo que el Estado debe priorizar la titulación de su territorio comunal, para así evitar la ocupación ilegal de su territorio.

Palabras clave: comunidades nativas, convenio 169 de la Organización Internacional del Trabajo (OIT), propiedad comunal

Abstract. The purpose of this research was to study the communal property right recognized in Convention 169 of the International Labor Organization (ILO) of the native communities of the Chazuta district, province and region of San Martín. The research is of an applied type of mixed quantitativequalitative approach, the non-experimental design of a cross-sectional

Citar como: Bardales del Aguila, L., \& Cenepo Mozombite, B. (2021). El derecho de propiedad comunal en el marco del Convenio 169 de la Organización Internacional del Trabajo (OIT) en las comunidades nativas del distrito de Chazuta, Provincia y Región de San Martin. Revista Científica Ratio lure, 1(1), 15-31. https://doi.org/10.51252/rcri .v1i1.106

Recibido: $15 / 11 / 2020$

Revisado: $15 / 12 / 2020$

Publicado: 31/01/2021 descriptive level, the population and study sample were made up of 64 inhabitants and 4 Apus and from the Kechwas native communities of Mushukllacta de Chipaota, Shilcayo, Canayo, Llucanayacu; located in the district of Chazuta, in the Lower Huallaga basin. To collect the information, two questionnaires were used: a survey for the residents and a structured interview for the Apus. The results of the surveys applied to the inhabitants of the four Communities show that $61 \%$ of the inhabitants do not know the amount of hectares belonging to the communal territory; Likewise, only $39 \%$ of the community members surveyed express that the State provides them with the necessary support for the titling of their territory, the Apus of the Communities of Shilcayo and Canayo, affirm that their territory is concessioned or superimposed on the Cordillera Escalera Regional Conservation Area and Cordillera Azul National Protection Forest. It is evident that the historical lags of the historical conflict due to the occupation and in many cases usurpation of the territory of the native peoples still persist, so the State must prioritize the titling of its communal territory, in order to avoid the illegal occupation of the territory.

Keywords: communal property, convention 169 of the International Labor Organization (ILO), native communities 


\section{$1 \quad$ Introducción}

Según el Ministerio de Cultura del Perú, "los pueblos indígenas u originarios son aquellos que tienen su origen en tiempos anteriores al Estado, y que, además, conservan todas o parte de sus instituciones distintivas. En el Perú habitan actualmente 55 pueblos indígenas u originarios. De ellos, 51 son originarios de la Amazonía y 4 de los Andes".

La investigación materia de estudio, es relevante y pertinente; porque, en la década presente se han acentuado los conflictos entre las Comunidades nativas de la Amazonia peruana; en el que se ven involucrados; el Estado, las Empresas privadas y población migrante (colonos) por la ocupación de los territorios de las comunidades nativas, el otorgamiento de concesiones de su territorio con fines de exploración y explotación, petrolera, minera, forestal y agraria; que en la mayoría de los casos se aplican sin tener en cuenta los parámetros normativos, acuerdos y convenciones suscritas entre el Estado y los organismos internaciones sobre los Derechos Humanos de la población indígena como: El Convenio 169 de la OIT, la Declaración Universal de los Derechos de los Pueblos Indígenas, la Convención Americana de los Derechos Humanos, y la Constitución Política.

Desde la Constitución Política de 1920, el Perú reconoce la existencia legal de los pueblos indígenas; sin embargo, en la práctica estos enfrentan una serie de dificultades para proteger sus territorios debido a la falta de un mecanismo eficiente que reconozca sus derechos sobre estos considerando la especial relación con su entorno y las formas tradicionales de organización y manejo de los mismos. La conceptualización y su reconocimiento, como pueblo indígena y comunidad nativa; se analiza los alcances de la existencia legal a que se refiere el artículo 14 del Convenio 169 de la OIT y los fundamentos de la propiedad comunal. (OIT, 2014).

Este trabajo busca contribuir al mejor entendimiento de la relación jurídica del indígena amazónico con su territorio ancestral y a la función social de la propiedad comunal. La investigación se desarrolló en cuatro comunidades nativas del distrito de Chazuta: Mushukllacta de Chipaota, Shilcayo, Canayo y Llucanayacu.

\subsection{Los pueblos indígenas en el marco del convenio 169 de la OIT}

El tratamiento dado por el derecho internacional a las poblaciones indígenas fue inicialmente realizado por la (OIT), entidad creada en 1919 en el marco de las negociaciones del tratado de Versalles que dieron origen a la Sociedad de las Naciones, órgano antecesor de las Naciones Unidas. (Aylwin, Carmona, Meza-Lopehandía, Silva \& Yáñez, 2009).

El Convenio $\mathrm{N}^{\circ} 169$ por la OIT como el primer tratado internacional de derechos humanos íntegramente referido a los pueblos indígenas dado en el marco de la evolución experimentada en

Citar como: Bardales del Aguila, L., \& Cenepo Mozombite, B. (2021). El derecho de propiedad comunal en el marco del Convenio 169 de la Organización Internacional del Trabajo (OIT) en las comunidades nativas del distrito de Chazuta, Provincia y Región de San Martin. Revista Científica Ratio lure, 1(1), 1531. https://doi.org/10.51252/rcri.v1i1.106 
el tratamiento de dichos pueblos y de sus derechos en el Sistema de Naciones Unidas. (Aylwin, Carmona, Meza-Lopehandía, Silva \& Yáñez, 2009).

En efecto, las perspectivas políticas y filosóficas dominantes a la época de su creación (1945), llevaron a la Organización de Naciones Unidas (ONU) a dar prioridad a la protección de los derechos y libertades individuales por sobre los derechos colectivos. Consecuente con ello, la ONU; enfrentó las violaciones y/o restricciones de derechos de personas debido a una característica de grupo (raza, origen étnico o nacional, o cultura), protegiendo los derechos de los individuos a través del principio de la no discriminación. (Aylwin, Carmona, Meza-Lopehandía, Silva \& Yáñez, 2009).

\subsection{El Convenio $\mathrm{N}^{\circ} 169$ de la OIT y la Declaración de las Naciones Unidas sobre los Derechos de los Pueblos Indígenas}

El Convenio No 169 fue aprobado en Perú por el Congreso Constituyente Democrático (CCD) de 1993, mediante Resolución Legislativa № 26253. El depósito (registro) internacional se hizo el 02 de febrero de 1994, adquiriendo por tanto vigencia desde el 02 de febrero de 1995. Tanto en virtud de la normativa vigente al momento de su ratificación (Constitución de 1979, artículo 105) como en la vigente en la actualidad (Constitución de 1993, artículo 55), y dada su condición de Tratado Internacional de Derechos Humanos, se entiende incorporado al ordenamiento con nivel constitucional según lo ha establecido el propio Tribunal Constitucional Peruano.

El Convenio 169 de la OIT, protege y reconoce el derecho a la tierra y territorio de los pueblos indígenas; en su Artículo 13.1. establece no sólo el reconocimiento material de territorio, sino que, incluye la cultura y los valores espirituales; asimismo, precisa en los artículos 15 y 16, el concepto de término tierra incluye el de territorio y el art. 14, prescribe" Deberá reconocerse a los pueblos interesados el derecho de propiedad y de posesión sobre las tierras que tradicionalmente ocupan".

De igual forma, la Declaración de las Naciones Unidas sobre los Derechos de los Pueblos Indígenas (DNUDPI) señala lo siguiente:

Artículo $26^{\circ}$. - 1) Los pueblos indígenas tienen derecho a las tierras, territorios y recursos que tradicionalmente han poseído, ocupado, utilizado o adquirido. 2) Los pueblos indígenas tienen derecho a poseer, utilizar y controlar las tierras, territorios y recursos que poseen en razón de la propiedad tradicional u otro tipo tradicional de ocupación o utilización, así como aquellos que hayan adquirido de otra forma. 3) Los Estados asegurarán el reconocimiento y protección jurídicos de estas tierras, territorios y recursos. Dicho reconocimiento respetará debidamente las costumbres, las tradiciones y los sistemas de tenencia de la tierra de los pueblos indígenas de que se traten.

Citar como: Bardales del Aguila, L., \& Cenepo Mozombite, B. (2021). El derecho de propiedad comunal en el marco del Convenio 169 de la Organización Internacional del Trabajo (OIT) en las comunidades nativas del distrito de Chazuta, Provincia y Región de San Martin. Revista Científica Ratio lure, 1(1), 1531. https://doi.org/10.51252/rcri.v1i1.106 
Por su parte, la Jurisprudencia de la Corte Interamericana de los Derechos Humanos (CIDH), en el caso del pueblo Saramaka vs. Surinam, Sentencia del 28 de noviembre de 2007. (Serie C N ${ }^{\circ}$ 172, párrafos 120-122) establece:

90.- Las decisiones de la Corte al respecto se han basado en la relación especial que los integrantes de los pueblos indígenas y tribales tiene con su territorio y en la necesidad de proteger su derecho a eses territorio, a fin de garantizar la supervivencia física y cultural de dichos pueblos ... 120.Sobre este particular, la Corte, ha sostenido, previamente, que ña subsistencia cultural y económica de los pueblos indígenas y túrbales y, por lo tanto, de sus integrantes, depende del acceso a y el uso de a los recursos naturales de su territorio, que están relacionados con su cultura y que se encuentran allí.

En el caso de la comunidad Mayagna (Sumo) Awas Tingni vs. Nicaragua, sobre Fondo de reparaciones y costas. Sentencia de 31 de agosto de 2011. Serie $C^{\circ} N^{\circ} 79$, párrafo 149:

$149^{\circ}$. (...) Para las comunidades indígenas, la relación con la tierra no es meramente una cuestión de posesión y producción, sino un elemento material y espiritual del que deben gozar plenamente, inclusive para preservar su legado cultural y transmitirlo a las generaciones futuras".

Es preciso mencionar que, el artículo 55 de la Constitución Política del Perú, establece que los tratados internacionales forman parte del derecho nacional. Y la Cuarta Disposición Final y Transitoria sostiene que los derechos se interpretan de conformidad con los tratados internacionales de derechos humanos; por lo que los incumplimientos de dichas normas constituyen faltas a una obligación internacional del Estado de proteger los derechos ancestrales de los pueblos indígenas. (Asociación Interétnica de Desarrollo de la Selva Peruana - AIDEISEP, 2019).

Como puede observarse, el Derecho internacional; tutela en toda su amplitud el derecho a la tierra y al territorio de las comunidades nativas; incluyendo el conocimiento, la espiritualidad, los recursos naturales como medio de subsistencia, obligando al Estado a salvaguardarlos y otorgar la garantía jurídica sobre dichos territorios.

Dureyttere (2003), precisa:

El derecho a la tierra y los recursos naturales no se refiere a la tierra sólo como medio deproducción y sustento económico sino, lo que es más importante, como territorio que define el espacio cultural y social necesario para la sobrevivencia física y cultural del grupo.

\subsection{Revisión del tratamiento de la legislación sobre la propiedad comunal}

El acercamiento al tema del derecho de propiedad debe hacerse a través de la revisión de las normas legales emitidas por el Estado. Primero la legislación sobre la propiedad de la comunidad

Citar como: Bardales del Aguila, L., \& Cenepo Mozombite, B. (2021). El derecho de propiedad comunal en el marco del Convenio 169 de la Organización Internacional del Trabajo (OIT) en las comunidades nativas del distrito de Chazuta, Provincia y Región de San Martin. Revista Científica Ratio lure, 1(1), 1531. https://doi.org/10.51252/rcri.v1i1.106 
campesina, partiendo del abordaje constitucional, y luego la forma como son tratados los derechos de los comuneros sobre la tierra. (Eguren F, Del Castillo L, Burneo Z \& Wiener E, 2008)

Conforme a nuestra actual Constitución (que reitera lo que las constituciones de 1920, 1933 y de 1979 señalaban) el Estado peruano garantiza el derecho de propiedad de las comunidades campesinas y de las comunidades nativas sobre la tierra. La propiedad comunal es una de las varias formas de ejercicio del derecho de propiedad que cuenta con la protección del Estado. El artículo 88 de la Constitución vigente dice que el Estado: "Garantiza el derecho de propiedad sobre la tierra, en forma privada o comunal o en cualquiera otra forma asociativa" (el énfasis es nuestro). (Eguren F, Del Castillo L, Burneo Z \& Wiener E, 2008)

La Constitución de 1979 reiteró las características del régimen de protección de las tierras comunales, esto es, que eran inalienables, inembargables e imprescriptibles, pero explicitó dos excepciones a la inalienabilidad (la decisión de la comunidad y la expropiación por el Estado) al señalar: (Eguren F, Del Castillo L, Burneo Z \& Wiener E, 2008, p.34)

Coincidente con las normas constitucionales entonces vigentes, el Código Civil de 1984 se ocupó de las tierras de las comunidades campesinas y nativas en su artículo 136, disponiendo que "Las tierras de las comunidades son inalienables, imprescriptibles e inembargables, salvo las excepciones establecidas por la Constitución Política del Perú”.

En 1987, se promulgan dos leyes especiales, relativas a las comunidades campesinas: La Ley General de Comunidades Campesinas, $n^{\circ} 24656$ y la Ley de Deslinde y Titulación del Territorio Comunal, $\mathrm{N}^{\circ}$ 24657, la primera; dispuso en su artículo 7, en concordancia con la Carta constitucional de 1979:

Asimismo, se ocupó de definir cuáles eran las tierras comunales, distinguiendo entre las originarias (que incluyen las tierras eriazas), las adquiridas por el derecho común y el derecho agrario y las adjudicadas por Reforma Agraria. Dicha Ley estableció un procedimiento administrativo sumamente expeditivo para la obtención de títulos por las comunidades campesinas que carecieran de los mismos o en caso que ellos tuvieran alguna discrepancia con la realidad. El proceso solo pasaba a los juzgados para la definición del área sobre la cual no pudiera establecerse con claridad el derecho de propiedad de la comunidad. (Eguren F, Del Castillo L, Burneo Z \& Wiener E, 2008, p.35)

\subsection{Jurisprudencia del Tribunal Constitucional (Exp. $\mathbf{N}^{\circ}$ 01126-2011-HC/TC)}

El caso seguido por Juana Griselda Payaba Cacique, presidenta de la Comunidad Nativa (Shipibo) Tres Islas, contra la intromisión en el ámbito de su territorio de madereros y mineros informales e ilegales que en Primera instancia y en la Sala Superior Mixta y de Apelaciones de la Corte

Citar como: Bardales del Aguila, L., \& Cenepo Mozombite, B. (2021). El derecho de propiedad comunal en el marco del Convenio 169 de la Organización Internacional del Trabajo (OIT) en las comunidades nativas del distrito de Chazuta, Provincia y Región de San Martin. Revista Científica Ratio lure, 1(1), 1531. https://doi.org/10.51252/rcri.v1i1.106 
Superior de Justicia de Madre de Dios, que declaró improcedente la demanda de agravio constitucional, recurriendo a la Sala Mixta y Penal de Apelaciones de la Corte Superior de Justicia de Lima; instancia que vuelve a declarar improcedente la demanda por los motivos expuestos anteriormente. Por lo que la demandante decide recurrir al Tribunal Constitucional.

El TC, falla y resuelve: 1) Declarar fundada la demanda de autos en lo que respecta a la afectación del derecho a la propiedad de la tierra comunal y del derecho a la autonomía comunal de la Comunidad Nativa Tres Islas. En consecuencia, NULA la Resolución N. ${ }^{\circ} 8$, de fecha 25 de agosto de 2010, derivada del Expediente N..$^{\circ}$ 00624-2010-0-2701-JR-PE-01, expedida por la Sala Mixta y Penal de Apelaciones de la Corte Superior de Justicia de Madre de Dios. 2) ORDENA a la Sala Mixta y Penal de Apelaciones de la Corte Superior de Justicia de Madre de Dios emita una nueva Resolución conforme a los fundamentos de la presente sentencia y, 3) ORDENA que cesen los actos de violación del territorio de la propiedad comunal y de autonomía de la Comunidad Nativa Tres Islas vinculados a este caso.

Al respecto el Tribunal Constitucional en el párrafo 22 del mencionado expediente establece:

22. Y si bien la constitución hace referencia a la protección de las tierras de las comunidades campesinas y nativas [artículo $88^{\circ}$ y $89^{\circ}$ de la Constitución], sin recoger el concepto de territorio de forma expresa, el Convenio 169 establece en su artículo $13^{\circ}$ que la utilización del término 'tierras' debe incluir el concepto de 'territorios'; la diferencia entre el concepto de tierra y territorio radica en que el primero se encuentra dentro de una dimensión civil o patrimonial, mientras que el segundo tiene una vocación política de autogobierno y autonomía. Así, esta dimensión política del término territorio se ajusta a la realidad de los pueblos indígenas, que descienden de las poblaciones que habitaban lo que ahora es el territorio de conquista y colonización, mantienen sus instituciones sociales, económicas, culturales y políticas, o partes de ellas.

\section{Materiales y métodos}

La investigación es de tipo aplicada de enfoque mixto cuantitativo-cualitativo; porque, para recoger la información correspondiente al conocimiento sobre la situación de las comunidades nativas de Mushukllacta de Chipaota, Shilcayo, Canayo y Llucanayacu, pertenecientes al distrito de Chazuta (Bajo Huallaga), Provincia y Región San Martín; en lo referente al proceso de reconocimiento y titulación de la propiedad y territorio comunal, se aplicó una encuesta, cuyos resultados fueron procesados cuantitativamente a través de la tabla de frecuencias y una entrevista estructurada aplicada a los 4 Apus de dichas comunidades nativas. Ambos instrumentos se orientan a identificar la percepción de pobladores y Apus sobre la problemática de la integridad

Citar como: Bardales del Aguila, L., \& Cenepo Mozombite, B. (2021). El derecho de propiedad comunal en el marco del Convenio 169 de la Organización Internacional del Trabajo (OIT) en las comunidades nativas del distrito de Chazuta, Provincia y Región de San Martin. Revista Científica Ratio lure, 1(1), 1531. https://doi.org/10.51252/rcri.v1i1.106 
y salvaguarda del derecho a la propiedad comunal ocupado ancestralmente por la población originaria asentada desde antes de la colonización europea.

La población y muestra de estudio la conforman 64 Pobladores de las Comunidades nativas, tomándose a 16 pobladores de cada comunidad a quienes se les aplicó una encuesta conformada por un cuestionario de 11 preguntas; cuyos resultados se presentan en 11 tablas de frecuencia y 4 Apus; a quienes, se les aplicó una entrevista con 11 preguntas, cuyo análisis cualitativo y cuantitativo se resume en una tabla resumen.

El diseño de la investigación fue no experimental, de tipo descriptivo transeccional; porque, se orientó a recoger información sobre hechos ocurridos en el medio natural con una sola medición, sin manipulación de variables. El procedimiento para la ejecución de la investigación, se desarrolló en tres (03) etapas:

Etapa I: Gabinete inicial. - Se seleccionó el tema y se realizó la verificación de Antecedentes, se caracterizó la realidad problemática, elaborándose la Justificación, Hipótesis y Variables, el Marco Teórico (Dispersión Temática) y Cuestiones Administrativas.

Etapa II: Trabajo de Campo. - Se aplicaron los instrumentos de recolección de datos entrevistándose a cuatro Apus y 48 pobladores de las comunidades, para identificar el nivel de conocimiento sobre el derecho de propiedad comunal, se solicitó documentación a la SUNARP para conocer si las comunidades nativas (Mushukllacta de Chipaota, Shilcayo, Canayo, Llucanayacu) del distrito de Chazuta cuentan con personería jurídica.

Etapa III: Gabinete final. - Se analizó y procesó la información y datos recogidos; cuyos resultados se presentan en tablas de frecuencia y gráficos. Finalmente se redactó el Informe de tesis, recomendando a los organismos competentes para la solución del problema de la vulneración del derecho de propiedad comunal de las Comunidades nativas de la región San Martín y de la Amazonía peruana.

\section{Resultados y discusiones}

Los Resultados de la encuesta aplicada a los pobladores de las Comunidades Nativas: Mushuk Llacta de Chipaota, Shilcayo, Canayo y Llucanayacu, se presentan en tablas que a continuación se presentan:

Citar como: Bardales del Aguila, L., \& Cenepo Mozombite, B. (2021). El derecho de propiedad comunal en el marco del Convenio 169 de la Organización Internacional del Trabajo (OIT) en las comunidades nativas del distrito de Chazuta, Provincia y Región de San Martin. Revista Científica Ratio lure, 1(1), 1531. https://doi.org/10.51252/rcri.v1i1.106 
Tabla 1

Conocimiento de la cantidad aproximada de hectáreas de tierras que pertenecen a la comunidad

\begin{tabular}{lcccc}
\hline \multirow{2}{*}{ COMUNIDAD } & \multicolumn{2}{c}{ SI } & \multicolumn{2}{c}{ NO } \\
\cline { 2 - 5 } & f & \% & f & \% \\
\hline Mushuk Llacta de Chipaota & 15 & 94 & 1 & 6 \\
Shilcayo & 9 & 56 & 7 & 44 \\
Canayo & 6 & 38 & 10 & 63 \\
Llucanayacu & 9 & 56 & 7 & 44 \\
TOTAL & $\mathbf{3 9}$ & $\mathbf{6 1}$ & $\mathbf{2 5}$ & $\mathbf{3 9}$ \\
\hline
\end{tabular}

Fuente: Elaboración propia

Interpretación: En la Tabla 1 se observa que 39 comuneros encuestados que representan el $61 \%$ respondieron que SI CONOCE cuántas hectáreas aproximadamente del territorio pertenecen a la comunidad, 35 comuneros, el 39\% respondió NO CONOCE. El porcentaje más alto de conocimiento corresponde a la Comunidad Mushuk Llacta de Chipaota; siendo la comunidad con menor conocimiento la de Canayo; ya que, de los 16 encuestados, el 63\% manifestó no tener conocimiento, lo propio ocurre con las comunidades de Shilcayo y Llucanayacu, en el que se manifiesta un $44 \%$ de desconocimiento.

Tabla 2

Conocimiento si el territorio comunal tiene los linderos establecidos

\begin{tabular}{lcccc}
\hline \multirow{2}{*}{ COMUNIDAD } & \multicolumn{2}{c}{ SI } & \multicolumn{2}{c}{ NO } \\
\cline { 2 - 5 } Mushuk Llacta de Chipaota & f & $\boldsymbol{\%}$ & f & $\boldsymbol{\%}$ \\
\hline Shilcayo & 16 & 25 & 0 & 0 \\
Canayo & 9 & 14 & 7 & 11 \\
Llucanayacu & 10 & 16 & 6 & 9 \\
TOTAL & 13 & 20 & 3 & 5 \\
\hline
\end{tabular}

Interpretación: Se observa que (48) encuestados, correspondiente al 75\% respondieron que, SI CONOCE que el territorio comunal tiene los linderos establecidos, el 25\% manifiesta no conoce. El porcentaje más alto de desconocimiento se presenta al $7 \%$ y $6 \%$ en las comunidades de Shilcayo y Canayo, mientras que la Comunidad con $0 \%$ es la de Mushuk Llacta de Chipaota.

Tabla 3

Cantidad de hectáreas proporcionadas por la comunidad para ser trabajadas con su familia

\begin{tabular}{lccccccccc}
\hline \multirow{2}{*}{ Hectáreas asignadas } & \multicolumn{2}{c}{$\begin{array}{c}\text { Mushuk Llacta de } \\
\text { Chipaota }\end{array}$} & \multicolumn{2}{c}{ Shilcayo } & \multicolumn{2}{c}{ Canayo } & \multicolumn{2}{c}{ Llucanayacu } \\
\cline { 2 - 9 } & $\mathrm{f}$ & $\%$ & $\mathrm{f}$ & $\%$ & $\mathrm{f}$ & $\%$ & $\mathrm{f}$ & $\%$ \\
\cline { 2 - 9 } $\mathbf{1 0}$ a 30 hectáreas & 6 & 38 & 0 & 0 & 9 & 56 & 13 & 81 \\
30 a 60 hectáreas & 5 & 31 & 12 & 75 & 5 & 31 & 3 & 19 \\
60 a 100 hectáreas & 1 & 6 & 3 & 19 & 1 & 6 & 0 & 0 \\
100 a más hectáreas & 4 & 25 & 1 & 6 & 1 & 6 & 0 & 0 \\
TOTAL & $\mathbf{1 6}$ & $\mathbf{1 0 0}$ & $\mathbf{1 6}$ & $\mathbf{1 0 0}$ & $\mathbf{1 6}$ & $\mathbf{1 0 0}$ & $\mathbf{1 6}$ & $\mathbf{1 0 0}$ \\
\hline
\end{tabular}

Citar como: Bardales del Aguila, L., \& Cenepo Mozombite, B. (2021). El derecho de propiedad comunal en el marco del Convenio 169 de la Organización Internacional del Trabajo (OIT) en las comunidades nativas del distrito de Chazuta, Provincia y Región de San Martin. Revista Científica Ratio lure, 1(1), 1531. https://doi.org/10.51252/rcri.v1i1.106 
Interpretación: En la tabla se observa que 28 comuneros $(37.50 \%)$ respondieron que la comunidad les otorgó 10 a 30 hectáreas para ser trabajadas, así mismo 25 comuneros (31.25\%) aseveraron que la comunidad les otorgó de 30 a 60 hectáreas, por consiguiente 5 comuneros (25\%) aseguraron que la comunidad les cedió entre 60 a 100 has y 6 comuneros indican que les fueron concedidas más de 100 hectáreas.

Tabla 4

Utilización de tierras asignadas a su familia

\begin{tabular}{|c|c|c|c|c|c|c|}
\hline \multirow{2}{*}{ Uso de tierras } & \multicolumn{2}{|c|}{$\begin{array}{l}\text { Sembríos } \\
\text { agrícolas }\end{array}$} & \multicolumn{2}{|c|}{ Conservación } & \multicolumn{2}{|c|}{ Otros } \\
\hline & $\mathbf{f}$ & $\%$ & f & $\%$ & f & $\%$ \\
\hline Mushuk Llacta de Chipaota & 12 & 75 & 2 & 13 & 2 & 13 \\
\hline Shilcayo & 14 & 88 & 2 & 13 & 0 & 0 \\
\hline Canayo & 10 & 63 & 5 & 31 & 1 & 6 \\
\hline Llucanayacu & 12 & 75 & 3 & 19 & 1 & 6 \\
\hline Total & 48 & 75 & 12 & 19 & 4 & 6 \\
\hline
\end{tabular}

Interpretación: La tabla muestra que, el $75 \%$ que representa a 48 comuneros; respondieron que las tierras se dedican para sembríos agrícolas; el 19\%, lo dedican para conservación y 4 que representan el $6 \%$ para otros fines. La comunidad de Canayo es la que manifiesta mayor porcentaje (31\%) de dedicación para Conservación. En lo referido a otros, se refieren a tierras dedicadas para pastos, en el que figuran 2 (13\%) comuneros de Mushuk Llacta de Chipaota, con cero $(0 \%)$ de áreas para pastos figura la comunidad de Shilcayo.

Tabla 5

Alguna vez el territorio comunal ha sufrido invasión por inmigrantes

\begin{tabular}{lcccc}
\hline \multirow{2}{*}{ COMUNIDAD } & \multicolumn{2}{c}{ SI } & \multicolumn{2}{c}{ NO } \\
\cline { 2 - 5 } & $\mathrm{f}$ & $\%$ & $\mathrm{f}$ & $\%$ \\
\hline Mushuk Llacta de Chipaota & 10 & 63 & 6 & 38 \\
Shilcayo & 10 & 63 & 6 & 38 \\
Canayo & 12 & 75 & 4 & 25 \\
Llucanayacu & 11 & 69 & 5 & 31 \\
TOTAL & $\mathbf{4 3}$ & $\mathbf{6 7}$ & $\mathbf{2 1}$ & $\mathbf{3 3}$ \\
\hline
\end{tabular}

Interpretación: En la tabla se observa que 43 comuneros encuestados (67\%) aseveraron que Sí han sufrido alguna invasión por inmigrantes, de los cuales 21 comuneros (33\%) contestaron que No han sido invadidos. Las Comunidades de Mushuk Llacta de Chipaota y Shilcayo presentan un mayor porcentaje de invasión, seguidos por Llucanayacu y Canayo

Citar como: Bardales del Aguila, L., \& Cenepo Mozombite, B. (2021). El derecho de propiedad comunal en el marco del Convenio 169 de la Organización Internacional del Trabajo (OIT) en las comunidades nativas del distrito de Chazuta, Provincia y Región de San Martin. Revista Científica Ratio lure, 1(1), 1531. https://doi.org/10.51252/rcri.v1i1.106 


\section{Tabla 6}

Conocimiento si el estado apoya el proceso de reconocimiento de las tierras otorgadas y del territorio comunal

\begin{tabular}{lcccc}
\multirow{2}{*}{ COMUNIDAD } & \multicolumn{3}{c}{ SI } & \multicolumn{2}{c}{ NO } \\
\cline { 2 - 5 } & $\mathrm{f}$ & $\%$ & $\mathrm{f}$ & $\%$ \\
\hline Mushuk Llacta de Chipaota & 2 & 3 & 14 & 22 \\
Shilcayo & 4 & 6 & 12 & 19 \\
Canayo & 6 & 9 & 10 & 16 \\
Llucanayacu & 13 & 20 & 3 & 5 \\
TOTAL & $\mathbf{2 5}$ & $\mathbf{3 9}$ & $\mathbf{3 9}$ & $\mathbf{6 1}$ \\
\hline
\end{tabular}

Interpretación: De la tabla se observa que 39 comuneros que representan el 61\% de encuestados confirmaron que el Estado No apoya el proceso de reconocimiento de las tierras y de su territorio, 25 comuneros que representa el 39\% confirmaron que Sí el estado viene apoyando el proceso de reconocimiento de las tierras otorgadas y al territorio de la comunidad. Los comuneros de Mushuk Llacta de Chipaota, Shilcayo y Canayo reconocen los porcentajes más bajos de apoyo estatal; el porcentaje más alto de reconocimiento lo manifiestan los comuneros de Llucanayacu $(20 \%)$.

Resultados de las entrevistas realizadas al APU de las comunidades nativas: Mushuk Llacta de Chipaota, Shilcayo, Canayo y Llucanayacu

Pregunta 1: ¿Conoce cuánto es el área (en hectáreas) aproximado del territorio que pertenece a su comunidad?

Respuestas: El APU de la comunidad nativa de Mushuk Llacta de Chipaota, respondió que si conoce y cuentan con 5872 hectáreas con título y resolución, además de encontrarse terrenos que aún están en ampliación. Los Apus de las comunidades de Shilcayo, Canayo y Llucanayacu; manifiestan no conocer ni cuentan con datos precisos sobre el área del territorio comunal.

Pregunta 2: ¿Conoce usted si el territorio de la comunidad tiene los linderos establecidos?

Respuestas: El APU de la comunidad nativa de Mushuk Llacta de Chipaota manifestó que su territorio tiene los linderos establecidos. El APU de la Comunidad de Canayo, precisó que lo tiene en parte: que por un lado con Santa Rosa de Chipaota y el Parque Cordillera Azul, faltando con Callanayacu. Los APUs de las comunidades de Shilcayo y Llucanayacu; manifiestan no conocen ni tienen los linderos establecidos.

Pregunta 3: ¿De qué manera los miembros de su comunidad ingresaron a ocupar el territorio que hoy ostentan?

Citar como: Bardales del Aguila, L., \& Cenepo Mozombite, B. (2021). El derecho de propiedad comunal en el marco del Convenio 169 de la Organización Internacional del Trabajo (OIT) en las comunidades nativas del distrito de Chazuta, Provincia y Región de San Martin. Revista Científica Ratio lure, 1(1), 1531. https://doi.org/10.51252/rcri.v1i1.106 
Respuestas. - Los cuatro APUs de las comunidades Mushuk Llacta de Chipaota, Shilcayo, Canayo y Llucanayacu, respondieron que el territorio se les otorgó por herencia.

Pregunta 4: ¿Desde cuándo viene ocupando el territorio comunal?

Respuestas: El APU de la comunidad nativa de Mushuk Llacta de Chipaota, indicó que lo vienen ocupando desde muchos años atrás y que fueron sus antepasados quienes nos legaron los territorios. Por su parte el APU de la comunidad de Shilcayo, menciona desde el año 1960, el de Canayo desde al año 1971 y Llucanayacu; desde el año 1965.

Pregunta 5: ¿Conoce usted si los territorios comunales vienen sufriendo constantes invasiones por inmigrantes?

Respuestas: Los Apus de las comunidades nativas de Mushuk Llacta de Chipaota y Canayo respondieron que si existen personas u inmigrantes que atentan o invaden nuestros territorios. Los Apus de las comunidades de Shilcayo y Llucanayacu; manifestaron no conocer ni registran casos de invasión de su territorio por migrantes.

Pregunta 6: ¿Qué acciones realizando ustedes frente a las invasiones de sus territorios por parte de los inmigrantes?

Respuestas: Los Apus de las comunidades nativas de Mushuk Llacta de Chipaota y Canayo respondieron que en este caso constatamos quien les faculta invadir el territorio, para luego comunicar a las autoridades competentes, luego desalojamos. Los Apus de las comunidades de Shilcayo y Llucanayacu; no haber desarrollado ninguna acción por no registrar la invasión de su territorio.

Pregunta 7: ¿El territorio comunal se encuentra inscrito en registros públicos, de ser afirmativa su respuesta, a quien corresponde su titularidad?

Respuesta: Los cuatro APUs de las comunidades Mushuk Llacta de Chipaota, Shilcayo, Canayo y Llucanayacu, respondieron que, Si está inscrito en la SUNARP, directamente a nombre de la comunidad. En el caso de la comunidad de Canayo; precisa el registro es de la Comunidad, pero no del territorio.

Pregunta 8: ¿El territorio comunal se encuentra dentro del área de Conservación Regional o dentro de la zona de conservación Nacional?

Respuestas: Los APUs de las comunidades de Mushuk Llacta de Chipaota y Llucanayacu Respondieron que No está dentro ni del Área de Conservación Regional ni dentro de la Zona de Conservación Nacional. Por su parte, los APUs de Shilcayo y Canayo; manifiestan que sí sus territorios se encuentran dentro del Área de Conservación Regional Cordillera Escalera.

Citar como: Bardales del Aguila, L., \& Cenepo Mozombite, B. (2021). El derecho de propiedad comunal en el marco del Convenio 169 de la Organización Internacional del Trabajo (OIT) en las comunidades nativas del distrito de Chazuta, Provincia y Región de San Martin. Revista Científica Ratio lure, 1(1), 1531. https://doi.org/10.51252/rcri.v1i1.106 
Pregunta 9: ¿Conoce usted, la existencia de áreas concesionadas que forman parte del territorio de su comunidad?

Respuestas: El APU de la comunidad de Mushuk Llacta de Chipaota, indicó que existían áreas concesionadas que ya fueron desalojados. El de la Comunidad de Shilcayo indica sólo el de la Área de Conservación Regional Cordillera Escalera. El APU de Canayo Respondió que Sí, el Parque Nacional Cordillera Azul. Y el de Llucanayacu manifiesta no conocer de área concesionada.

Pregunta 10: El estado en la actualidad viene apoyando con la protección y conservación del área que ocupa su comunidad?

Respuestas: Los APUs de las Comunidades de Mushuk Llacta de Chipaota, Canayo y Llucanayacu, manifestaron que actualmente ya no existe ningún apoyo por parte del estado con la protección y conservación del área que ocupa la comunidad. Solamente el APU de Shilcayo indicó: que el estado Sí les apoya con la protección y conservación del área que ocupa su comunidad.

Pregunta 11: ¿Si un miembro de la comunidad quiere disponer del predio que viene conduciendo, puede hacerlo?

Respuestas: El APU de la Comunidad de Mushuk Llacta de Chipaota que sólo puede vender su área trabajado, es decir con plantaciones. En cambio, los APUs de las Comunidades de Shilcayo, Canayo y Llucanayacu, respondieron que No puede hacerlo ya que está prohibido la venta del territorio dentro de la comunidad. El APU de Llucanayacu recalca que este acto es castigado.

\section{Discusión}

La Constitución Política del Perú, en su art. 89 reconoce la existencia legal de las comunidades nativas, allí precisa la realización de procedimientos administrativos para que la comunidad acceda al reconocimiento e inscripción de su personería jurídica y para el reconocimiento de sus derechos de propiedad sobre los territorios comunales que estos pueblos ocupan. Ortega (2014) afirmó: de la revisión de los títulos de 11 comunidades del Río Ene (entre ellas el de la C.N Samaniato) y 28 comunidades del Río Tambo, pudo comprobar que primero se reconoce la existencia de una "comunidad nativa" y luego la propiedad del territorio que esta posee.

La mayoría de estos títulos se han logrado por el esfuerzo de las propias organizaciones indígenas con el apoyo de Organizaciones No Gubernamentales (ONGs), que a través de convenios y con financiamiento de la cooperación internacional, consiguieron los fondos para financiar estos procesos, realizando un contraste con la presente investigación las comunidades nativas de

Citar como: Bardales del Aguila, L., \& Cenepo Mozombite, B. (2021). El derecho de propiedad comunal en el marco del Convenio 169 de la Organización Internacional del Trabajo (OIT) en las comunidades nativas del distrito de Chazuta, Provincia y Región de San Martin. Revista Científica Ratio lure, 1(1), 1531. https://doi.org/10.51252/rcri.v1i1.106 
Mushukllacta de Chipaota, Shilcayo, Canayo, Llucanayacu del distrito de Chazuta, cuentan con personería jurídica, pero sin embargo hasta el momento no cuentan con la propiedad de sus territorios, conllevando a diversos conflictos, principalmente por la invasión de migrantes, que de diferentes formas buscan aprovecharse de sus tierras que por más de 50 años vienen habitando, cuidando y protegiendo, así mismo ninguna organización viene apoyando en el tedioso proceso del respeto a su derecho comunal, conllevando a la vulneración del derecho de propiedad comunal establecido en el marco normativo del Convenio 169 de la OIT.

Ortega (2014) afirmó: las políticas de titulación de tierras en la Amazonía tienen una clara tendencia a facilitar la titulación individual de predios y un mínimo de interés en fortalecer los sistemas de tenencia comunal y manejo sostenible de recursos que vienen aplicando las comunidades Asháninka del Río Tambo desde tiempos inmemoriales. Consideramos que el desarrollo de estas políticas ha tenido impactos negativos (tanto sociales como ambientales) para los pueblos indígenas. Así mismo el presente estudio muestra que las comunidades nativas en el distrito de Chazuta tienen una representación de una población vulnerable, siendo que con el transcurrir de los años mediante una ardua lucha se ha logrado reconocer sus derechos como tales, pero hasta la actualidad se sigue evidenciando falencias en el respeto del derecho de propiedad, es así que en la encuesta realizada un porcentaje mayor del $80 \%$ afirmaron que no poseen ningún documento que sustente la entrega del territorio comunal por parte del Apu, así mismo un porcentaje alto arrojó que más del $60 \%$ no conoce en que consiste la titulación comunal, estos motivos se deben a que hasta la actualidad se vienen transgrediendo el derecho de propiedad comunal.

Ruiz (2012) refiere que este derecho fundamental tiene su origen en el Convenio 169 de la OIT, el cual constituye una norma internacional que reconoce a los pueblos indígenas, pero en nuestro país la legislación especial no se ha implementado de manera correcta, si bien es cierto es un proceso, no una charla informativa o audiencia pública, existiendo relación directa con el presente resultado, ya que hasta la actualidad por más de 50 años de ser consideradas como comunidades, se sigue evidenciando la falta de cumplimiento del derecho de propiedad comunal.

Souza (2015), señala que los pueblos indígenas poseen derechos colectivos e individuales y uno de ellos es el derecho cultural a la autodeterminación, a la dignidad, los cuales en su protección garantizan la autonomía y diversidad cultural. Siendo así, los derechos fundamentales requieren de una protección muy especial desde un aspecto particular y colectivo a cada caso en concreto. En el presente estudio se investigó a la propiedad comunal como un derecho fundamental, que además de estar normado es de mucha relevancia su aplicación, que vela por la protección de las formas de vida, regidas por la comunidad, en este contexto, este derecho es poco respetado por las diferentes instituciones y poblaciones migrantes, que día a día invaden sus territorios, 
conllevando así a la pérdida de sus tradiciones, identidad cultural y territorio nativo. Guevara (2011) señala que en nuestro país la diversidad de culturas hace que se tenga que respetar la coexistencia e interacción de los pueblos dentro de cada territorio. Su aplicación debe ser en conjunto con el principio de flexibilidad teniendo en cuenta las circunstancias de cada lugar.

Angles (2016), sostiene que existen muchos proyectos de inversión, de diferentes naturaleza o actividad de explotación de los recursos naturales, pero la gran mayoría de estos no respetan el derecho de propiedad comunal, debido a que la legislación no cuenta con una institución fiscalizadora y además aprovechan los vacíos legales y deficiencias de la ley, en tal sentido no se da una correcta aplicación. Cabe señalar que su escasa aplicación se debe a que en diversas ocasiones se realiza con la intención de favorecer a personas con predios privados, con ello se hace evidente la poca importancia del respeto por la propiedad comunal y la no valoración de la diversidad cultural en las comunidades de Mushukllacta de Chipaota, Shilcayo, Canayo, Llucanayacu.

\section{Conclusiones}

Se viene vulnerando el derecho de propiedad comunal reconocido en el Convenio 169 de la Organización Internacional del Trabajo (OIT) en las comunidades nativas del distrito de Chazuta, que se da a través de la inexistencia de linderos establecidos, presencia de migrantes asentadas en territorios de comunidades nativas, desconocimiento de la población de las comunidades nativas sobre el derecho de propiedad comunal, falta de apoyo y voluntad política por parte del estado y funcionarios, falta de empoderamiento por parte de la comunidad, lideres (as) y por funcionarios, además de la ausencia de celeridad en procedimientos iniciados por la comunidad para la titulación comunal.

Todas las comunidades nativas del distrito de Chazuta cuentan con personería jurídica, Mushukllacta de Chipaota desde el año 2013, Shilcayo y Canayo en el año 2006, Llucanayacu en el año 2019.

El nivel de conocimiento de los comuneros y Apus demostró que el 53\% tiene conocimiento sobre el derecho de propiedad comunal, sin embargo, un porcentaje alto de $47 \%$ no conoce ni tiene conocimiento alguno, siendo este uno de los factores que conllevan a la vulneración de sus derechos como comunidades nativas.

Los Apus de: Mushuk Llacta de Chipaota, Canayo y Llucanayacu manifiestan que el Estado muestra poco interés para el apoyo y conservación del territorio comunal a excepción del APU de la comunidad de Shilcayo. Asimismo, afirman que parte de su territorio se encuentra expuesto a las concesiones como el del Área de Conservación Regional Cordillera Escalera, el Parque Nacional Cordillera Azul y la presencia de población migrante, a los que se ven obligados a

Citar como: Bardales del Aguila, L., \& Cenepo Mozombite, B. (2021). El derecho de propiedad comunal en el marco del Convenio 169 de la Organización Internacional del Trabajo (OIT) en las comunidades nativas del distrito de Chazuta, Provincia y Región de San Martin. Revista Científica Ratio lure, 1(1), 1531. https://doi.org/10.51252/rcri.v1i1.106 
desalojarlos por atentar contra la integridad de su territorio y por ende la biodiversidad y su medio de subsistencia.

\section{Recomendaciones}

Se recomienda desarrollar nuevas propuestas que permitan a las comunidades nativas del distrito de Chazuta, el reconocimiento de sus derechos sobre su territorio ancestral, vía adecuados programas de saneamiento, demarcación y titulación de tierras y territorios indígenas, basados en el respeto por las formas de tenencia comunal de los indígenas.

Recomendamos un análisis mucho más profundo sobre la situación de tenencia de la tierra en las comunidades Mushukllacta de Chipaota, Shilcayo, Canayo, Llucanayacu y que involucre además a un mayor número de comunidades de la Región San Martín, especialmente aquellas directamente vinculadas al Parque Nacional Cordillera Azul, bosque de Protección Alto Mayo, área de Conservación Regional Cordillera Escalera y Parque Nacional Río Abiseo.

Se recomienda fortalecer los sistemas de tenencia comunal y manejo sostenible de recursos que vienen aplicando las comunidades Mushukllacta de Chipaota, Shilcayo, Canayo, Llucanayacu desde tiempos inmemoriales. Consideramos que el desarrollo de estas políticas ha tenido impactos negativos (tanto sociales como ambientales) para los pueblos indígenas.

La creación y/o actualización de un catastro de comunidades en la Región San Martín, debido a que se ha ido ampliando la frontera agrícola y ocasionando con ello un impacto negativo en las comunidades debido al uso inadecuado de los territorios y recursos. Es por ello que es urgente la implementación de la Ley 282949 que crea el Sistema Nacional Integrado de Catastro y su vinculación con el Registro de Predios; esta Ley ha sido reglamentada por Decreto Supremo $\mathrm{N}^{\circ} 005-2006-J U S$ del 10 de febrero de 2006.

Se recomienda a los organismos competentes del Estado como: MINISTERIO DE AGRICULTURA para realizar la supervisión y control in situ de las diferentes actividades agropecuarias ilegales por migrantes, a la SUNARP en facilitar la inscripción de los actos y derechos de las comunidades nativas, a través de procedimientos sencillos, flexibles y prácticos y a la DIRECCIÓN REGIONAL AGRARIA en acelerar el proceso en el envío del Título de Propiedad y plano correspondiente a los Registros Públicos de la Provincia en la cual se encuentran asentadas las Comunidades.

Agradecimiento a la Universidad Nacional de San Martín-Tarapoto, por haber financiado en presente Proyecto de Investigación.

Citar como: Bardales del Aguila, L., \& Cenepo Mozombite, B. (2021). El derecho de propiedad comunal en el marco del Convenio 169 de la Organización Internacional del Trabajo (OIT) en las comunidades nativas del distrito de Chazuta, Provincia y Región de San Martin. Revista Científica Ratio lure, 1(1), 1531. https://doi.org/10.51252/rcri.v1i1.106 


\section{Referencias bibliográficas}

Asociación Interétnica de Desarrollo de la Selva Peruana (AIDESEP) y Derecho y Ambiente y Recurso Naturales (DAR) (2019). Informe Jurídico de salvaguardas y garantías para la protección de Pueblos Indígenas en Situación de Aislamiento y en Situación de Contacto Inicial (PIACI) de la Reserva Territorial Kugapakori, Nahua, Nanti y otros (RTKNN). Lima. DAR.

Angles, G. (2016). El Contenido Constitucional del Derecho a la Consulta Previa. Recuperado de: http://blog.pucp.edu.pe/blog/derechoconstitucionalperu/2015/05/21/el-contenidoconstitucional-del-derecho-a-la-consulta-previa/

Aylwin, Carmona, Meza-Lopehandía, Silva \& Yáñez (2009). Las implicancias de la Ratificación del Convenio $\mathrm{N}^{\circ} 169$ de la OIT en Chile. Disponible en: https://www.goredelosrios.cl/cultura2/wp-content/uploads/2016/02/Las-Implicanciasde-la-Ratificaci\%C3\%B3n-del-Convenio-N\%C2\%B0-169-de-la-OIT-en-ChileJos\%C3\%A9-Aylwin-y-otros.pdf

Aylwin, J. (2002). El Derecho de los Pueblos Indígenas a la Tierra y al Territorio en América Latina: Antecedentes Históricos y Tendencias Actuales. Washington, D.C. - Salón Simón Bolívar.

Banco Interamericano de Desarrollo. (2014). Evaluación comparativa: Proyectos de regularización y administración de tierras. Oficina de evaluación y supervisión.

Bonilla T., W. A. (2015). Derechos de los pueblos originarios Mayangna y Miskitu a la propiedad comunal y a la justicia. Programa de doctorado en Paz Conflicto y Democracia. Granada: Instituto de Paz y Conflicto.

Deruyttere, A. (2003). Pueblos indígenas, recursos naturales y desarrollo con identidad: Riesgos y oportunidades en tiempos de globalización. Disponible en: https://publications.iadb.org/es/pueblos-indigenas-recursos-naturales-y-desarrollo.

Eguren F, Del Castillo L, Burneo Z \& Wiener E. (2008). Los derechos de propiedad sobre la tierra en las comunidades campesinas. CEPES. Disponible en: http://www.cies.org.pe/sites/default/files/.

Guevara, A. (2011). Las causas estructurales de la pluralidad legal en el Perú". En Revista Derecho y Sociedad. Lima, Año II, Nº XVI, pp. 325-340.

Martínez, C. (1986). Comunidades, pueblos y naciones indígenas. Naciones Unidas.

Ministerio de Agricultura y Riego. (2014). Ministerio de Agricultura y Riego. Obtenido de http://www.minagri.gob.pe/portal/especial-iv-cenagro/70-marco-legal/titulacionagraria-en-el-peru/414-el-pett

Ministerio de Cultura (2014). Derechos de los pueblos indígenas en el Perú. El rol garante del estado en la protección y promoción de los derechos humanos. Obtenido en: https://centroderecursos.cultura.pe/sites/default/files/rb.

Oficina de Evaluación y Supervisión (OVE) (2014). Proyectos de Regularización y Administración de Tierras. Banco Interamericano de Desarrollo. Disponible en: file:///C:/Users/Usuario/Downloads/Proyectos-de-Regularizaci\%C3\%B3n.

Ortega, N. (2014). El Derecho de Propiedad Comunal Indígena en la Amazonía y su Regulación en la Legislación Peruana. Lima. Perú. Disponible en: http://tesis.pucp.edu.pe/repositorio/handle/.

Citar como: Bardales del Aguila, L., \& Cenepo Mozombite, B. (2021). El derecho de propiedad comunal en el marco del Convenio 169 de la Organización Internacional del Trabajo (OIT) en las comunidades nativas del distrito de Chazuta, Provincia y Región de San Martin. Revista Científica Ratio lure, 1(1), 1531. https://doi.org/10.51252/rcri.v1i1.106 
Ruiz, J. (2012). La Consulta Previa de los Pueblos Indígenas En El Perú. Análisis y Comentarios de cada artículo de La Ley De Consulta Previa y su Reglamento. (1ra.Ed.) Lima: Instituto de Defensa Legal.

Souza, R. (2015), Pueblos indígenas, diversidad cultural y el derecho cultural a la autodeterminación: desde el derecho internacional al Constitucionalismo Latinoamericano. Artículo científico publicado en la Pontificia Universidad Católica del Perú.

Tribunal Constitucional (2012). Exp. $\quad \mathrm{N}^{\circ}$ 01126-2011-HC/TC. Disponible en: https://tc.gob.pe/jurisprudencia/2012/01126-2011-HC.html

Yrigoyen, R. (2004). Pluralismo jurídico, derecho indígena y jurisdicción especial en los países andinos. Disponible en: https://www.cejamericas.org/Documentos.

\section{Contribuciones de los autores}

El Bachiller Cenepo Mozombite Basilio, contribuyó con el trabajo de campo, aplicación de las encuestas y contacto con la población de estudio, Apus y moradores de las comunidades nativas.

El Dr. Lionel Bardales del Aguila, contribuyó con el procesamiento de la información y redacción del artículo científico

Citar como: Bardales del Aguila, L., \& Cenepo Mozombite, B. (2021). El derecho de propiedad comunal en el marco del Convenio 169 de la Organización Internacional del Trabajo (OIT) en las comunidades nativas del distrito de Chazuta, Provincia y Región de San Martin. Revista Científica Ratio lure, 1(1), 1531. https://doi.org/10.51252/rcri.v1i1.106 